\title{
Analysis of the expression of human tumor antigens in ovarian cancer tissues
}

\author{
Rouba Ali-Fehmi ${ }^{a, c}, 1$, Madhumita Chatterjee ${ }^{\mathrm{b}, 1}$, Alexei lonan ${ }^{\mathrm{b}}$, Nancy K. Levin ${ }^{\mathrm{b}}$, Haitham \\ Arabi $^{\mathrm{a}}$, Sudeshna Bandyopadhyay ${ }^{\mathrm{a}}$, Jay P. Shah ${ }^{\mathrm{c}}$, Christopher S. Bryant ${ }^{\mathrm{C}}$, Stephen M. \\ Hewitt $^{\mathrm{d}}$, Michael G. O'Rand ${ }^{\mathrm{e}}$, Oleg M. Alekseev ${ }^{\mathrm{e}}$, Robert Morris ${ }^{\mathrm{c}}$, Adnan Munkarah ${ }^{\mathrm{c}, f}$, Judith \\ Abrams $^{\mathrm{g}}$, and Michael A. Tainsky ${ }^{\mathrm{a}, \mathrm{b}}{ }^{*}{ }^{*}$ \\ aDepartment of Pathology, Wayne State University School of Medicine, Detroit, MI 48201, USA \\ bProgram in Molecular Biology and Human Genetics, Karmanos Cancer Institute/Wayne State \\ University, 110 E. Warren, Detroit, MI 48201, USA \\ 'Division of Gynecologic Oncology, Wayne State University School of Medicine, Harper \\ Professional Office Bldg, 4160 John R., 2nd Floor, Detroit, MI 48201, USA \\ dTissue Array Research Program, Laboratory of Pathology, Center for Cancer Research, National \\ Cancer Institute, NIH, MSC 4605 Bethesda, MD 20892-4605, USA \\ eDepartment of Cell and Developmental Biology, University of North Carolina, Chapel Hill, 27599, \\ USA \\ fDivision of Gynecologic Oncology, Henry Ford Health System, Department of Women's Health \\ Services, 2799 W. Grand Boulevard, Detroit, MI 48202, USA (current address) \\ gBarbara Ann Karmanos Cancer Institute and Wayne State University, 428 HWCRC, 4100 John \\ R, Detroit, MI 48201, USA
}

\begin{abstract}
Biomarkers for early detection of cancer have great clinical diagnostic potential. Numerous reports have documented the generation of humoral immune responses that are triggered in response to changes in protein expression patterns in tumor tissues and these biomarkers are referred to as tumor associated antigens (TAAs). Using a high-throughput technology, we previously identified 65 proteins as diagnostically useful TAAs by profiling the humoral immune responses in ovarian cancer (OVCA) patients. Here we determined the expression status of some of those TAAs in tissues from OVCA patients. The protein expression patterns of 4 of those 65 antigens, namely NASP, RCAS1, Nijmegen breakage syndrome1 (NBS1) and eIF5A, along with p53 and Her2 (known molecular prognosticators) and two proteins that interact with NBS1, MRE11 and RAD50, were assessed by immunohistochemistry (IHC). NASP and RCAS1 proteins were more frequently expressed in ovarian cancer tissues than with normal ovarian tissue and serous cystadenomas and MRE11 was less frequently expressed. When evaluated simultaneously, only NASP and MRE11 remained statistically significant with sensitivity of $66 \%$ and specificity of $89 \%$. None of these proteins' expression levels were prognostic for survival. Together, our results indicate that occurrence of humoral immune responses against some of these TAAs in OVCA patients is triggered by antigen protein overexpression.
\end{abstract}

\footnotetext{
(C) 2009/2010 - IOS Press and the authors. All rights reserved

*Corresponding author. Tel.: +1 313578 4340; Fax: +1 313832 7294; tainskym@karmanos.org.

${ }^{1}$ Both should be considered first authors.
} 


\section{Keywords}

Ovarian cancer; phage display; tissue biomarker; humoral immune response; tissue microarray

\section{Introduction}

Epithelial ovarian cancer is the eighth most common malignancy in the United States, with nearly 15,000 deaths annually [20]. Screening for ovarian cancer has been based on strategies using serum tumor markers or ultrasound imaging of the ovaries. A recent report from Menon et al. indicates that specificity of multimodal screening (annual CA125 screening with transvaginal ultrasound (TVS) as a second-line test) is higher than annual screening with TVS alone for all primary epithelial ovarian and tubal cancers [28]. However, CA125 is elevated in only about $50 \%$ of patients with clinically detectable early stage OVCA [26]. Therefore, there is a need for new technologies to identify a panel of biomarkers that can be used in a non-invasive screening test that has both high sensitivity and high specificity in the heterogeneous population of ovarian cancer patients.

Proteomic technologies have proved useful in the field of discovery of tumor tissue biomarkers. Candidate tissue biomarkers of ovarian tumors are often derived from studies of overexpression of mRNAs that were previously found to be up-regulated in ovarian cancer by analyses of gene expression [18]. Many tumor antigens have been reported to be overexpressed (at protein level) in OVCA; these include cell cycle proteins [27], cancer testis antigens like MAGE- A4 and NY-ESO-1 [49]. TAAs can also be identified in studies of proteins that are shed from cancer cells into the circulation [25] and can form blocking factors with the antibodies that may help tumors escape immune surveillance [45].

It has been reported that numerous intracellular proteins can elicit humoral immune responses in different cancer patients [44] as a result of aberrant expression of antigen biomarkers [7], alternative splicing of pre-messenger RNAs [47], point mutations [48] and over-expression [10] or post-translation alterations of the expressed antigens such as changes in glycosylation [15], phosphorylation [12]. Proteomic approaches that are based on the idea of using serum antibody responses to different autoantigens are multifaceted and may provide more reliable serum markers for diagnosis of cancer $[32,38]$.

In searching for a more robust method for the early detection of OVCA, we chose to identify large numbers of potential diagnostic antibodies in serum and developed a high-throughput strategy to clone antigen biomarkers. We refer to this approach as "epitomics" [13]. Because antibodies to any single antigen tend to detect only a small fraction of individuals with cancer, we recognized the necessity to screen a large panel of potential antigen markers. Therefore, we used a differential biopanning technique to screen $\mathrm{T} 7$ phage display cDNA libraries to isolate cDNAs coding for epitopes binding to antibodies present specifically in the sera of patients with early or late stage OVCA but not binding with antibodies in the sera of healthy women. Protein microarray-based immunoassays of 480 phage clones obtained by differential biopanning of a T7 phage OVCA cDNA library with sera obtained from cancer patients, patients with benign gynecological diseases and healthy females resulted in the identification of 65 antigens [6]. The statistical validation of these 65 biomarkers was then conducted on a different array platform using independent serum samples. The objective of this study was to determine the association of the expression patterns of a subset of those 65 OVCA antigens that we identified through epitomics with 1) the generation of humoral immune responses in OVCA patients and, 2) the clinical characteristics of OVCA in patients' tumor tissues. We evaluated the protein expression levels of a panel of 4 antigens namely, NASP, RCAS1, NBS1, eIF5A, along with 2 known prognostic factors p53 
and Her2 and two other proteins MRE11 and RAD50 that interact with NBS1 (the MRN complex), by immunohistochemical staining of tumor specimens of paraffin-embedded tissues and tissue microarrays obtained from women who had epithelial ovarian cancer at a variety of stages and histotypes. We found that some epitomics antigens were overexpressed in OVCA relative to normal or benign ovarian tissues thus indicating that overexpression of those antigens resulted in their immunogenicity. Over-expression of NASP effectively discriminated OVCA from healthy or benign tissues. None of the biomarkers distinguished healthy tissues from benign or non-serous tumors from serous tumors; however, RCAS1 did distinguish early stage from late stage OVCA. None of the markers provided prognostic information relevant to overall survival.

\section{Materials and methods}

\subsection{Patients}

This study was open to women newly diagnosed with OVCA or who were within five years of initial diagnosis or a recurrence occurring between 1997 and 2005 (Cohort I). We used formalin fixed, paraffin embedded tissue sections (FFPE) from 126 women who underwent primary surgery and received no chemotherapy or radiation therapy prior to their surgery (Median age $=57, \mathrm{IQR}=49-67$ ). The normal control group included 20 patients who had undergone a hysterectomy and bilateral salpingo-oophorectomy due to uterine leiomyoma (Median age $=49$, IQR $=44-60)$. Another control group included 20 women who had benign ovarian serous cystadenoma (Median age $=52, \mathrm{IQR}=42-66)($ Table $1 \mathrm{~A})$. Blocks were obtained from the Detroit Medical Center/Barbara Ann Karmanos Cancer Institute Pathology Core Facility and the Gynecologic Oncology Group Tissue Bank (GOG). H\&E stained slides for all cases and controls were reviewed for confirmation of diagnosis and histology by the study pathologist (RA-F); only cases with serous, mucinous, endometrioid, or clear cell histology were utilized. An alternative classification strategy developed by Shih and Kurman was also employed (Figs 5-6) [40]. In this model all low-grade serous, mucinous, endometrioid, or clear cell carcinoma surface epithelial ovarian tumors are designated as Type I; high-grade serous carcinoma is designated as Type II so as to distinguish indolent, low-grade ovarian neoplasms from high-grade lesions that behave far more aggressively, yet appear to have no precursor lesion.

Because of missing data on vital status and follow-up, we were unable to assess the predictive utility of the markers on survival in this cohort. We therefore evaluated survival using a tissue microarray constructed by the Karmanos Cancer Institute Pathology Core Facility (Cohort II) in a cohort comprised of patients who were diagnosed with epithelial ovarian carcinoma between 1985 and $2004(n=200)$ (Table 1B). Demographic data and information on surgical treatment was obtained from a retrospective review of medical records. Survival data were retrieved using the institutional Clinical Information System and the Metropolitan Detroit Cancer Surveillance System Database (MDCSS), a participant in the National SEER Registry. Surgical staging was determined using the criteria recommended by International Federation of Gynecology and Obstetrics (FIGO). Histologic type and grade were determined using previously described World Health Organization criteria. Tissue microarrays were prepared from FFPE blocks using a manual tissue arrayer (MTA-1, Beecher Instruments, Sun Prairie WI). A single block was selected per case and from each block, three $1.5 \mathrm{~mm}$ diameter cores were obtained [2,34].

All protocols were approved by the Wayne State University Human Investigation Committee. A waiver of consent was obtained for the retrospective review of archived material. 
We also evaluated an additional cohort using independent tissue microarray provided by the Tissue Array Research Program (TARP) of the National Cancer Institute, National Institutes of Health, Bethesda, MD. The NCI TARP3 array consisted of 500 anonymized samples representing a variety of malignant and normal tissues: Healthy $(n=50)$, Brain Tumor $(n=$ 25), Breast Adenocarcinoma $(n=75)$, Colonic Adenocarcinoma $(n=75)$, Lung Cancer $(n=$ $75)$, Lymphoma $(n=50)$, Melanoma $(n=25)$, Ovarian Adenocarcinoma $(n=50)$, Prostatic Adenocarcinoma $(n=75)$. The FFPE tissue used to construct this array was provided by the Cooperative Human Tissue Network (CHTN).

\subsection{Immunohistochemistry}

The protein expression levels of NASP, RCAS1, NBS1, MRE11, RAD50, eIF5A, p53 and Her2 were measured by immunohistochemical staining of $4 \mu \mathrm{m}$ sections or tissue microarray (TMA) cores from FF-PE tissue. Staining was either carried out manually or by using the Ventana automated instrument, BenchMark- XT following manufacturer's instructions (Ventana Medical Systems, Inc., 1910 Innovation Park Drive, Tucson, AZ. 85737).

\section{Manual immunostaining using primary antibodies for Her2, NASP, NBS1 and} RCAS1-Each section was deparaffinized and subjected to immunohistochemical staining with standard streptavidinbiotin-peroxidase techniques, with diaminobenzidine as the chromogen.

\section{Automated immunostaining using primary antibodies for elF5A, p53, MRE11 and RAD50-Sections were deparaffinized with a solution of EZ-Prep (Ventana \#950-101), rinsed with reaction buffer (Ventana \#950-300) and treated with inhibitor solution (Ventana DAB detection kit \#760-2021) to decrease the endogenous peroxidase activity.}

Additional details for both manual and automated processes are listed in Table 2.

Two pathologists (RA-F and HA) who were blinded to the demographic and clinical information about the participants evaluated the tissue sections and cores under a light microscope. For evaluating the expression of the parameters, the areas of highest staining density were identified. For the expression of Her2 strong and complete membranous expression in greater than 5\% of the cells was defined as positive staining. Cytoplasmic expression of eIF5A and RCAS1 was considered positive when expressed in more than 5\% of the tumor cells. Nuclear expression was estimated to evaluate the expression of NASP, NBS1, RAD50, MRE11 and p53 and considered positive when expressed in more than 5\% of tumor cells after counting at least 1000 cells. If the percentage of stained cells was less than or equal to five percent, the case was considered negative, and if it exceeded five percent, the case was considered positive (Table 3(A-D)).

Tissue microarray-The TARP3 TMA was read by SMH. p53 was scored as negative ( $5 \%$ tumor cells), or positive ( $>5 \%$ tumor cells). Her 2 was scored as 1,2 , or 3 (per standard Her2 protocol), eIF5A, NASP, and RCAS1 were scored as negative (no staining seen), weak (score 1), intermediate/moderate (score 2), or strong (score 3). For technical reasons we were not able to analyze protein expression of NBS, MRE11 and RAD50 on the TARP3 TMA.

\subsection{Statistical analyses}

Association between biomarkers was assessed with two-sided Fisher's exact tests. Association of age with biomarker expression was evaluated using Kruskal-Wallis tests. 
Discriminatory ability of the antigen biomarkers was assessed with logistic regression.

Survival times were computed from the date of diagnosis to the date of death or last followup. Cox regression models, controlling for age and stage were used to evaluate prognostic value of the markers for overall survival. Bootstrapping with 1000 replications of each model was used for internal validation. Significance levels are adjusted for multiple comparisons using Holm's method [17].

\subsection{Determination of mRNA level of biomarkers by quantitative real time pcr (Q-RT-PCR)}

Total RNA was isolated from the surgical tumor specimens obtained from patients with malignant serous ovarian carcinoma using RNeasy kit (Qiagen Inc., Valencia, CA, USA). Total RNA $(2 \mu \mathrm{g})$ was reversed transcribed into cDNA using Superscript II (Life Technologies, Gaithersburg, MD, USA). The quantitation of mRNA using Q-RT-PCR was performed following the methods as described previously [23].

\section{Results}

Humoral immunity resulting in the development of autoantibodies directed against TAAs is being employed as a potential diagnostic tool for early detection of cancer. Studies have shown that circulating antibodies can be detected long before there are clinical manifestations of cancer [24]. The humoral immune responses in cancer patients are related to overexpression of proteins i.e. Her2 [10], aberrant expression of mutated proteins or proteins with various post-translational modification in tumor cells $[11,19,39]$. In our previous study, which was mainly directed at the discovery of tumor biomarkers in serum, we profiled humoral immune responses elicited in OVCA patients [6]. The expression of a small subset of those previously identified 65 biomarkers was analyzed in this study to better understand the mechanism by which humoral immune responses are generated in OVCA patients and to determine whether TAAs have value as tissue biomarkers. IHC was performed at the protein level for 4 of these biomarkers to determine if their immunogenicity was due to overexpression.

\subsection{Immunohistochemical staining for tissue biomarkers}

Analyses were restricted to cases with serous, mucinous, endometroid, or clear cell histology (Table 1B). Among the 65 biomarkers identified by serum immunoscreening [6], we were able to acquire antibodies for expression studies of 4 biomarkers, NASP [1, 36], NBS1, RCAS1, and eIF5A. We also analyzed 2 known biomarkers of OVCA, p53 and Her2 (Table 2). Although MRE11 and RAD50 were not members of the group of 65 biomarkers, we assessed their expression because these proteins physically interact in cells with NBS1 at sites of DNA double strand breaks after the formation of a ternary complex. Eventually the MRE11/RAD50/NBS1 (MRN) complex gets recruited to the site of DNA double-strand breaks for DNA repair [21].

Overexpression of NASP protein was observed in $46 \%$ of patients with OVCA, in 5\% of patients with serous cystadenoma, and in 5\% normal ovaries ((Fig. 1A, 3A) and Table 3(AB)). Overexpression of RCAS1 protein was observed in $81 \%$ of late stage OVCA patients, in $50 \%$ of patients with serous cystadenoma, and in $20 \%$ of women with normal ovaries ((Fig. 1B, 3B) and Table 3(B-C)). Both NASP and RCAS1 provided good utility in discriminating cancer from healthy as described later. None of the other markers such as, eIF5A (Fig. 1C, 3C), NBS1 (Fig. 1D, 3D), MRE11 (Fig. 2A, 4A) and RAD50 (Fig. 2B, 4B) showed markedly higher expression in all OVCA patients compared to women with serous cystadenoma and those with normal ovaries (Table 3A, 3B). Although expression of MRE11 and RAD50 proteins was lower in OVCA patients than in the control groups (healthy ovary and serous cystadenoma), these markers did not adequately discriminate between control 
groups and cancer because the expression pattern of these antigens did not differ significantly between control groups and cancer. Among the known prognostic biomarkers, overexpression of Her2 protein was observed in 29\% of all OVCA patients, in $30 \%$ of serous cystadenoma patients, and in 5\% of women with normal ovaries ((Fig. 2C, 4C) and Table 3 (A-B)). Overexpression of p53 protein was observed in $45 \%$ of all OVCA patients, in none of serous cystadenoma patients, and in none of the normal ovaries ((Fig. 2D, 4D) and Table 3(A-B)). An alternative classification strategy was employed in which all lowgrade serous, mucinous, endometrioid, or clear cell carcinoma surface epithelial ovarian tumors were designated as Type I and high-grade serous carcinoma was designated as Type II [40]. All the markers except NBS1 retained their significance when tumors were classified as Type I, Type II rather than serous, non-serous (Figs 5-6).

Seventy six out of two hundred OVCA patients used for the immunoscreeningon protein microarrays for the identification of autoantigens were also used for IHC analysis to evaluate the expression of those antigens in the tumor specimens obtained from the same OVCA patients. However, we did not see a relationship between dye ratio (microarray analysis) and intensity $\mathrm{x} \%$ cells staining (IHC analysis) for the OVCA patients used in both the analyses.

\subsection{Diagnostic value of biomarkers}

When the markers were evaluated one at a time for their ability to discriminate between healthy ovaries and serous cystadenoma from ovarian cancer, we found that NASP ( $p<$ $0.001)$ and RCAS1 $(p=0.01)$ were significantly more likely to be positive in tissues from women with OVCA (Table 3A) and MRE11 $(p=0.01)$ was less likely to be positive. p53 could not be evaluated because none of the healthy ovaries or those with benign tumors expressed p53. With the exception of p53 $(p=0.02)$, (data not shown) there were no statistically significant differences in age between women who expressed the antigen marker and those who did not (all $p>0.50$ ).

When the markers were evaluated simultaneously, NASP $(p<0.001)$ and MRE11 $(p=$ $0.004)$ retained their significance, but RCAS1 did not $(p=0.22)$, which is probably due to the association between RCAS1 and NASP $(p<0.002)$, uncorrected for multiple comparisons). Using a 2 marker model, the probability of OV-CA is $57 \%$ for women who are negative for NASP and positive for MRE11; the probability of OVCA is 99\% for women who are positive for NASP and negative for MRE11. The probability of OVCA is $92 \%$ for women who are negative for both markers and $96 \%$ for women who are positive for both markers. If a positive test is defined as having a predicted probability greater than $90 \%$, this model has $66 \%$ sensitivity and $89 \%$ specificity and the area under the ROC curve is 0.78 .

When the markers were evaluated further one at a time (Table 3B), we found that only HER2 expression $(p=0.02)$ was differentially expressed between healthy ovaries and benign serous cystadenomas; in the model with all of the markers, none were statistically significant. NASP $(p=0.02), \operatorname{RCAS} 1(p=0.01)$ and $\operatorname{RAD50}(p=0.02)$ were found to be more common in women with late stage disease than in those with early stage disease in single marker models and RCAS1 $(p=0.04)$ remained a significant predictor of late stage disease when evaluated in a multivariable model with all of the other markers (Table 3C). $\operatorname{NASP}(p<0.001), \mathrm{p} 53(p=0.02), \operatorname{RAD50}(p=0.006)$ and NBS1 $(p<0.001)$ and RCAS1 $(p$ $=0.04)$ were more common in with women serous ovarian cancer than in those with nonserous disease in single marker models, but none were important discriminators in the multivariable model (Table 3D). The conclusions are the same when women are categorized as having Type I or Type II disease. 


\subsection{Utility of markers against other cancers}

Useful markers should not only distinguish healthy and benign ovaries from OVCA samples, but differentiate between ovarian and other cancers as well. We assessed utility of p53, NASP, Her2, eIF5A, and RCAS1 on tissue microarrays from samples with other cancers (see Materials and Methods). The best markers for classification of OVCA against other cancers were NASP $(\mathrm{SN}=64 \%, \mathrm{SP}=75 \%)$ and $53(\mathrm{SN}=32 \%, \mathrm{SP}=94 \%)$. Because one marker may be not sufficient for a good classifier, we assessed the utility of combination of p53 and NASP. Comparing OVCA with other cancers, sensitivity and specificity were $66 \%$ and $75 \%$ respectively. Because of the enhanced risk of breast and colon cancers along with OVCA in high-risk BRCA1 and BR-CA2 families, it was important to determine discriminatory accuracy of the tissue biomarkers for these cancers compared to OVCA [4]. Individually, NASP had the highest accuracy with $64 \%$ sensitivity for OVCA and specificity of $73 \%$ and $68 \%$ to breast and colon cancer respectively. If individuals are classified as positive if any of $\mathrm{p} 53$, Her2, and NASP are positive, sensitivity to distinguish OVCA from breast and colon cancer is $81 \%$, while specificity is $58 \%$ and $46 \%$ to breast and colon cancer respectively. Overall, NASP and p53 were the best markers at distinguishing OVCA from other cancers, but neither was adequate.

\subsection{Prognostic value for survival}

The prognostic value of the markers for survival was assessed using samples from 200 patients in cohort II (Table 1B). Approximately half (104/200) have died and median follow-up time for those still alive is 44 months. More than $75 \%$ of the women in cohort II expressed NASP, RCAS1, RAD50 or eIF5A (Table 4). Nearly 2/3rd expressed MRE11, almost half expressed NBS1 or p53 while $12 \%$ expressed Her2. When assessed for prognostic value after adjusting for the known prognostic factors age at diagnosis, stage of disease and whether the tumor grade was borderline or not, none of the individual markers nor the combination was significantly associated with survival.

\subsection{Correlative study of antigen expression at mRNA level (Q-RT-PCR) and protein level (IHC)}

To examine the relationship between expression at mRNA and protein level, we used fold change score for Q-RT-PCR data and percentage of cells stained positive for the biomarkers (NASP, RCAS1, NBS1, MRE11, RAD50, eIF5A, p53 and Her2) as assessed by IHC. NASP and MRE11 showed positive correlations between expression at mRNA and protein level: NASP Spearman rho $=0.68(p=0.02)$.

\section{Discussion}

The humoral immune response plays an important role in self-defense against various diseases including cancer. Numerous studies have reported the presence of circulating tumor reactive immunoglobulins (IgGs) in patients diagnosed with melanoma [29], breast [9], and OVCA [46]. The generation of antibodies directed against intracellular antigens, such as cMyc and p53, as a result of tissue destruction has been reported in cancer patients [5]. The humoral immune response can be induced by inappropriate expression or overexpression of self-antigens (silent or expressed at low level in normal tissues) [30] besides other mechanisms as discussed earlier. The occurrence of immunogenicity of self-antigens due to overexpression is based on the growing evidence that support the presence of self-reactive $\mathrm{T}$-cells in the T-cell repertoire that are able to recognize endogenous peptides derived from self-antigen. Studies have shown that only well-expressed self-antigen determinants are efficient in tolerizing T-cells. However, there are 'subdominant' or 'cryptic' determinants of self-antigens that are poorly processed from native molecule and inefficiently presented to $\mathrm{T}$ cells and do not induce such tolerance [8]. But when overexpression of self-antigen occurs 
in cancer, these cryptic determinants are presented to T-cells in context with MHC molecules in the presence of various co-stimulatory molecules in the tumor microenvironment and elicit immunological responses [31].

In our previous study, we established a new technology to find TAAs in OVCA patients by exploiting autoantibody signatures in OVCA patients. We identified 65 protein biomarkers by profiling humoral immune response in OVCA patients using high-throughputepitomics technology [6]. In order to determine the mechanism for the generation of immunogenicity of TAA biomarkers, we analyzed 4 of 65 antigens for which the antibodies were available for expression at the protein level. Immunohistochemical staining of tissue sections (obtained from OVCA patients) was performed to detect the expression profiles of NASP, RCAS1, NBS1, eIF5A (a subset of the 65 antigens), MRE11, RAD50, as well as p53 and Her2 (known prognostic biomarkers).

The IHC analysis revealed overexpression of NASP in OVCA patients when compared to serous cystadenoma patients or in normal ovary as shown in Table $3(\mathrm{AB})$. NASP is a histone H1-binding protein and provides efficient transport of histones into the nucleus during cell division cycle and helps in nucleosome remodeling during DNA replication [37]. As NASP is a cell cycle regulated protein, overexpression of NASP as indicated by our results may be involved in higher level of mitotic activity and cell proliferation in ovarian cancer as shown in other studies in Hela cells by Richardson et al. [36]. Oram et al. reported that testis specific protein Y-encoded (TSPY) gene, a histone binding protein involved in cell cycle regulation, was shown to potentiate cell proliferation and tumoregenesis in Hela and NIH3T3 cells [33]. Based on the above facts, a possible functional role of NASP in tumor progression in OVCA can be envisioned but further experimental investigation is necessary. On the other hand, RCAS1 showed overexpression only in the late stage serous OVCA patients when compared to serous cystadenoma patients or in normal ovary (Table 3 $(\mathrm{B}-\mathrm{C})$ ). RCAS1 is a membrane protein that is released into circulation by ectodomain shedding and is involved in the induction of apoptotic cell death of lymphocytes thereby allowing tumor to escape immune surveillance in cancer patients [41]. Previously, overexpression of RCAS1 in uterine and ovarian carcinomas was reported by Sonoda et al. [43] and their recent study indicated that a significantly higher concentration of serum RCAS1 in OVCA patients that suggested that RCAS1 may be involved in tumor progression in OVCA patients [42]. Thus, our results of overexpression of RCAS1 in OV-CA are in accordance with other published studies. Disis et al. reported a direct correlation between the degree of generation of humoral immune response and overexpression of Her2 in breast cancer patients [14]. Their study showed that the endogenous Her2-specific humoral immune response was greater in patients with Her2 overexpressing tumors than in patients who had lower levels of Her2 expression. Our previous study already had reflected the generation of immune responses against NASP and RCAS1 antigens because these antigens were identified on protein arrays when interrogated with IgGs from OVCA patients' sera [6]. In the current study, NASP, NBS1, RAD50 and RCAS1 as well as p53 were more commonly expressed in serous ovarian tumor specimens than in non-serous. Overall, this result indicates that overexpression of these TAAs may be related to the generation of humoral immune responses in OVCA patients. Interestingly, 76 of the OVCA patients used for the detection of autoantigens by immunoscreening on protein microarrays were also tested for protein expression analysis by IHC in the tumor tissue specimens obtained from the same OVCA patients. However, we did not observe any correlation between serum IgG level binding to that antigen on microarray analysis and the protein expression level of tumor antigens in IHC analysis. The observed humoral immune response may not always correlate with the overexpression of tumor antigens because of a number of reasons. First, serum antibodies are polyclonal in nature and some patients may have antibodies that are directed towards the cloned epitope of an antigen, while others may have antibodies 
exhibiting higher affinity for a different epitope other than the cloned one. Therefore, in these patients humoral immune response against that specific antigen may not be detectable on protein microarrays despite its over-expression. Second, the excess antigens shed from tumors can bind to their corresponding antibodies in the circulation thus interfering with the binding of T-cell receptors to an epitope of tumor antigens for which they are specific. These blocking factors may cause a lowering of serum IgGs for specific antigen in cancer patients [45]. Third, reports from different studies suggest that TAAs that are carried to the lymph nodes and are presented to T-cells by antigen presenting cells can drive clonal deletion [22] or anergy [3] in different tumor microenvironments of cancer patients.

The difference in levels of protein expression between women with OVCA and controls is not statistically significant for RCAS1, RAD50, Her2, NBS1, eIF5A and p53 (all $p \searrow 0.30$ ). The level of MRE11 protein expression is significantly lower $(p=-0.004)$ in OVCA patients than in controls and the levels of NASP $(p<0.001)$ is significantly higher. Based on the relative expression profiles of NBS1, MRE11 and RAD50 in ovarian tumors compared to serous cystadenomas or normal ovaries, our result may indicate the absence of an intact DNA double strand breaks repair pathway in OVCA patients. As MRN complex plays an integral role in cellular response to DNA double strands breaks, decreased MRN levels could be associated with progression in tumor development through less efficient DSB repair in combination with impaired checkpoint signaling and an apoptotic defect. As overexpression for NBS1, MRE11 and RAD50 was not observed in OVCA patients, the cause for their immunogenicity may be due to presence of mutations in the respective genes in cancer patients as reported from other studies. A report from Plisiecka-Halasa et al. indicated presence of NBS1 gene mutation in OVCA patients [35]. Mutations in MRE11 and RAD50 genes in breast cancer or OVCA was reported by Heikkinen et al. [16]. Genomic analysis for somatic mutations within these 3 genes was beyond the scope of the present study.

The expression of known prognostic markers like p53 and Her2 was also analyzed by IHC. p53 showed overexpression in OVCA patients compared to ovaries in serous cystadenoma patients. Although p53 was highly specific in distinguishing between OVCA and control group, this marker did not have prognostic utility as reported in previous studies. Other markers individually or in combination were not significant predictors of survival.

We assessed utility of markers to distinguish OVCA from the control non-cancer group individually, as a panel of markers, to distinguish between early and late stage, as well as specificity to OVCA against benign conditions and other cancers. Based on these analyses, NASP was more likely to be positive in all OV-CA, serous, early stage, and late stage OVCA tissues compared to non-cancer group. Moreover, NASP was more likely to be positive in serous compared to non-serous OVCA, and in OVCA compared to other cancers. Overall, this study shows that finding biomarkers with high-throughput epitomic technology is a promising new way to identify tissue biomarkers for cancer.

An evaluation of the associations between expression of antigens at protein and mRNA level was also performed in this study. A highly significant correlation between expression at mRNA and protein level was obtained for NASP (Spearman rho $=0.68(p=0.02)$ ). This result suggests that the expression of NASP may be regulated at the transcriptional level in these tissues. However, no significant associations were found for the other biomarkers. Overexpression of 6 out of 8 mR-NAs for these markers namely, NASP, eIF5A, NBS1, RAD50, Her2, and p53 in ovarian carcinoma compared to normal ovary was found in the Oncomine database of ovarian Oncomine Gene Expression Signatures. ${ }^{9}$

\footnotetext{
${ }^{9}$ Oncomine database is available at http://www.oncomine.org/ocm/main/main.jsp.
} 
In conclusion, by profiling humoral immune responses in OVCA patients we identified diagnostically useful TAAs using a protein microarray-based high-throughput technology. We demonstrated that overexpression of some of these TAAs at the protein level as assessed by IHC, may be related to the generation of their immunogenicity that elicited humoral immune responses in OVCA patients. Although identified for their diagnostic utility, these TAAs for early detection of OVCA in serum also have diagnostic potential as tissue biomarkers. This new panel of biomarkers will hold promise as potential tissue biomarkers that may have better therapeutic utility in a clinical setting of cancer screening and prevention.

\section{Acknowledgments}

We acknowledge Natalie Victor Shirley for coordinating the tissue samples, the Karmanos Cancer Institute Gynecologic Oncology support staff for assisting in identifying eligible cases and consenting them to the study and the Gynecologic Oncology Group Tissue Bank (GOG), which is funded by the National Cancer Institute. We thank Barbara Pruetz for performing immunohistochemical staining for all the biomarkers in tissue sections obtained from OVCA patients. This project was supported by funds from the Gail Purtan Ovarian Cancer Research Fund, and by grants from National Institutes of Health (NIH), R21/R33-CA100740 and U01-117748, and The Michigan Life Science Corridor Fund (085P300470). Production of anti-NASP antibodies was funded by a grant to MGO from the Eunice Kennedy Shriver NICHD cooperative agreement [U54 (HD35041)] as part of the Specialized Cooperative Centers Program in Reproduction and Infertility Research.

\section{References}

1. Alekseev OM, Bencic DC, Richardson RT, Widgren EE, O'Rand MG. Overexpression of the Linker histone-binding protein tNASP affects progression through the cell cycle. J Biol Chem. 2003; 278:8846-8852. [PubMed: 12509435]

2. Ali-Fehmi R, Che M, Khalifeh I, Malone JM, Morris R, Lawrence WD, Munkarah AR. The effect of cyclooxygenase-2 expression on tumor vascularity in advanced stage ovarian serous carcinoma. Cancer. 2003; 98:1423-1429. [PubMed: 14508829]

3. Appleman LJ, Boussiotis VA. T cell anergy and costimulation. Immunol Rev. 2003; 192:161-180. [PubMed: 12670403]

4. Brose MS, Rebbeck TR, Calzone KA, Stopfer JE, Nathanson KL, Weber BL. Cancer risk estimates for BRCA1 mutation carriers identified in a risk evaluation program. J Natl Cancer Inst. 2002; 94:1365-1372. [PubMed: 12237282]

5. Canevari S, Pupa SM, Menard S. 1975-1995 revised anti-cancer serological response: biological significance and clinical implications. Ann Oncol. 1996; 7:227-232. [PubMed: 8740784]

6. Chatterjee M, Mohapatra S, Ionan A, Bawa G, Ali-Fehmi R, Wang X, Nowak J, Ye B, Nahhas FA, Lu K, Witkin SS, Fishman D, Munkarah A, Morris R, Levin NK, Shirley NN, Tromp G, Abrams J, Draghici S, Tainsky MA. Diagnostic markers of ovarian cancer by high-throughput antigen cloning and detection on arrays. Cancer Res. 2006; 66:1181-1190. [PubMed: 16424057]

7. Chen YT, Scanlan MJ, Sahin U, Tureci O, Gure AO, Tsang S, Williamson B, Stockert E, Pfreundschuh M, Old LJ. A testicular antigen aberrantly expressed in human cancers detected by autologous antibody screening. Proc Natl Acad Sci USA. 1997; 94:1914-1918. [PubMed: 9050879]

8. Cibotti R, Kanellopoulos JM, Cabaniols JP, Halle-Panenko O, Kosmatopoulos K, Sercarz E, Kourilsky P. Tolerance to a self-protein involves its immunodominant but does not involve its subdominant determinants. Proc Natl Acad Sci USA. 1992; 89:416-420. [PubMed: 1370355]

9. Conroy SE, Sasieni PD, Fentiman I, Latchman DS. Autoantibodies to the 90kDa heat shock protein and poor survival in breast cancer patients. Eur J Cancer. 1998; 34:942-943. [PubMed: 9797714]

10. Disis ML, Knutson KL, Schiffman K, Rinn K, McNeel DG. Pre-existent immunity to the HER-2/ neu oncogenic protein in patients with HER-2/neu overexpressing breast and ovarian cancer. Breast Cancer Res Treat. 2000; 62:245-252. [PubMed: 11072789]

11. Disis ML, Pupa SM, Gralow JR, Dittadi R, Menard S, Cheever MA. High-titer HER-2/neu proteinspecific antibody can be detected in patients with early-stage breast cancer. J Clin Oncol. 1997; 15:3363-3367. [PubMed: 9363867] 
12. Dittmer F, Pohlmann R, von FK. The phosphorylation pattern of oligosaccharides in secreted procathepsin D is glycosylation site-specific and independent of the expression of mannose 6phosphate receptors. J Biol Chem. 1997; 272:852-858. [PubMed: 8995373]

13. Draghici S, Chatterjee M, Tainsky MA. Epitomics: serum screening for the early detection of cancer on microarrays using complex panels of tumor antigens. Expert Rev Mol Diagn. 2005; 5:735-743. [PubMed: 16149876]

14. Goodell V, Waisman J, Salazar LG, de la RC, Link J, Coveler AL, Childs JS, Fintak PA, Higgins DM, Disis ML. Level of HER-2/neu protein expression in breast cancer may affect the development of endogenous HER-2/neu-specific immunity. Mol Cancer Ther. 2008; 7:449-454. [PubMed: 18319334]

15. Hakomori S. Glycosylation defining cancer malignancy: new wine in an old bottle. Proc Natl Acad Sci USA. 2002; 99:10231-10233. [PubMed: 12149519]

16. Heikkinen K, Karppinen SM, Soini Y, Makinen M, Winqvist R. Mutation screening of Mre11 complex genes: indication of RAD50 involvement in breast and ovarian cancer susceptibility. J Med Genet. 2003; 40:e131. [PubMed: 14684699]

17. Holm S. A simple sequentially rejective multiple test procedure. Scand J Statist. 1979; 6:65-70.

18. Hough CD, Cho KR, Zonderman AB, Schwartz DR, Morin PJ. Coordinately up-regulated genes in ovarian cancer. Cancer Res. 2001; 61:3869-3876. [PubMed: 11358798]

19. Jager D, Unkelbach M, Frei C, Bert F, Scanlan MJ, Jager E, Old LJ, Chen YT, Knuth A. Identification of tumor-restricted antigens NY-BR-1, SCP-1, and a new cancer/testis-like antigen NW-BR-3 by serological screening of a testicular library with breast cancer serum. Cancer Immun. 2002; 2:5. [PubMed: 12747750]

20. Jemal A, Siegel R, Ward E, Murray T, Xu J, Smigal C, Thun MJ. Cancer statistics, 2006. CA Cancer J Clin. 2006; 56:106-130. [PubMed: 16514137]

21. Kobayashi J. Molecular mechanism of the recruitment of NBS1/hMRE11/hRAD50 complex to DNA double-strand breaks: NBS1 binds to gamma-H2AX through FHA/BRCT domain. J Radiat Res (Tokyo). 2004; 45:473-478. [PubMed: 15635255]

22. Kruisbeek AM, Amsen D. Mechanisms underlying T-cell tolerance. Curr Opin Immunol. 1996; 8:233-244. [PubMed: 8725947]

23. Kulaeva OI, Draghici S, Tang L, Kraniak JM, Land SJ, Tainsky MA. Epigenetic silencing of multiple interferon pathway genes after cellular immortalization. Oncogene. 2003; 22:4118-4127. [PubMed: 12821946]

24. Li Y, Karjalainen A, Koskinen H, Hemminki K, Vainio H, Shnaidman M, Ying Z, Pukkala E, Brandt-Rauf PW. p53 autoantibodies predict subsequent development of cancer. Int J Cancer. 2005; 114:157-160. [PubMed: 15523685]

25. Luftner D, Luke C, Possinger K. Serum HER-2/neu in the management of breast cancer patients. Clin Biochem. 2003; 36:233-240. [PubMed: 12810150]

26. Mann WJ, Patsner B, Cohen H, Loesch M. Preoperative serum CA-125 levels in patients with surgical stage I invasive ovarian adenocarcinoma. J Natl Cancer Inst. 1988; 80:208-209. [PubMed: 3162284]

27. Masciullo V, Scambia G, Marone M, Giannitelli C, Ferrandina G, Bellacosa A, Benedetti PP, Mancuso S. Altered expression of cyclin D1 and CDK4 genes in ovarian carcinomas. Int J Cancer. 1997; 74:390-395. [PubMed: 9291427]

28. Menon U, Gentry-Maharaj A, Hallett R, Ryan A, Burnell M, Sharma A, Lewis S, Davies S, Philpott S, Lopes A, Godfrey K, Oram D, Herod J, Williamson K, Seif MW, Scott I, Mould T, Woolas R, Murdoch J, Dobbs S, Amso NN, Leeson S, Cruickshank D, McGuire A, Campbell S, Fallowfield L, Singh N, Dawnay A, Skates SJ, Parmar M, Jacobs I. Sensitivity and specificity of multimodal and ultrasound screening for ovarian cancer, and stage distribution of detected cancers: results of the prevalence screen of the UK Collaborative Trial of Ovarian Cancer Screening (UKC-TOCS). Lancet Oncol. 2009; 10:327-340. [PubMed: 19282241]

29. Merimsky O, Shoenfeld Y, Chaitchik S, Yecheskel G, Fishman P. Antigens and antibodies in malignant melanoma. Tumour Biol. 1994; 15:188-202. [PubMed: 7939169] 
30. Naftzger C, Takechi Y, Kohda H, Hara I, Vijayasaradhi S, Houghton AN. Immune response to a differentiation antigen induced by altered antigen: a study of tumor rejection and autoimmunity. Proc Natl Acad Sci USA. 1996; 93:14809-14814. [PubMed: 8962137]

31. Nanda NK, Sercarz EE. Induction of anti-self-immunity to cure cancer. Cell. 1995; 82:13-17. [PubMed: 7606778]

32. Naora H, Yang YQ, Montz FJ, Seidman JD, Kurman RJ, Roden RB. A serologically identified tumor antigen encoded by a homeobox gene promotes growth of ovarian epithelial cells. Proc Natl Acad Sci USA. 2001; 98:4060-4065. [PubMed: 11274429]

33. Oram SW, Liu XX, Lee TL, Chan WY, Lau YF. TSPY potentiates cell proliferation and tumorigenesis by promoting cell cycle progression in HeLa and NIH3T3 cells. BMC Cancer. 2006; 6:154. [PubMed: 16762081]

34. Pansare V, Munkarah AR, Schimp V, Haitham AM, Saed GM, Morris RT, Ali-Fehmi R. Increased expression of hypoxia-inducible factor 1alpha in type I and type II endometrial carcinomas. Mod Pathol. 2007; 20:35-43. [PubMed: 17099695]

35. Plisiecka-Halasa J, nsonka-Mieszkowska A, Rembiszewska A, Bidzinski M, Steffen J, Kupryjanczyk J. Nijmegen breakage syndrome gene (NBS1) alterations and its protein (nibrin) expression in human ovarian tumours. Ann Hum Genet. 2002; 66:353-359. [PubMed: 12485469]

36. Richardson RT, Alekseev OM, Grossman G, Widgren EE, Thresher R, Wagner EJ, Sullivan KD, Marzluff WF, O'Rand MG. Nuclear autoantigenic sperm protein (NASP), a linker histone chaperone that is required for cell proliferation. J Biol Chem. 2006; 281:21526-21534. [PubMed: 16728391]

37. Richardson RT, Batova IN, Widgren EE, Zheng LX, Whitfield M, Marzluff WF, O'Rand MG. Characterization of the histone H1-binding protein, NASP, as a cell cycle-regulated somatic protein. J Biol Chem. 2000; 275:30378-30386. [PubMed: 10893414]

38. Scanlan MJ, Chen YT, Williamson B, Gure AO, Stockert E, Gordan JD, Tureci O, Sahin U, Pfreundschuh M, Old LJ. Characterization of human colon cancer antigens recognized by autologous antibodies. Int J Cancer. 1998; 76:652-658. [PubMed: 9610721]

39. Scanlan MJ, Gout I, Gordon CM, Williamson B, Stockert E, Gure AO, Jager D, Chen YT, Mackay A, O'Hare MJ, Old LJ. Humoral immunity to human breast cancer: antigen definition and quantitative analysis of mRNA expression. Cancer Immun. 2001; 1:4. [PubMed: 12747765]

40. Shih I, Kurman RJ. Ovarian tumorigenesis: a proposed model based on morphological and molecular genetic analysis. Am J Pathol. 2004; 164:1511-1518. [PubMed: 15111296]

41. Sonoda K, Miyamoto S, Hirakawa T, Yagi H, Yotsumoto F, Nakashima M, Watanabe T, Nakano H. Association between RCAS1 expression and microenvironmental immune cell death in uterine cervical cancer. Gynecol Oncol. 2005; 97:772-779. [PubMed: 15943986]

42. Sonoda K, Miyamoto S, Yotsumoto F, Yagi H, Nakashima M, Watanabe T, Nakano H. Clinical significance of RCAS1 as a biomarker of ovarian cancer. Oncol Rep. 2007; 17:623-628. [PubMed: 17273743]

43. Sonoda K, Nakashima M, Kaku T, Kamura T, Nakano H, Watanabe T. A novel tumor-associated antigen expressed in human uterine and ovarian carcinomas. Cancer. 1996; 77:1501-1509. [PubMed: 8608535]

44. Stockert E, Jager E, Chen YT, Scanlan MJ, Gout I, Karbach J, Arand M, Knuth A, Old LJ. A survey of the humoral immune response of cancer patients to a panel of human tumor antigens. $\mathrm{J}$ Exp Med. 1998; 187:1349-1354. [PubMed: 9547346]

45. Taylor DD, Black PH. Shedding of plasma membrane fragments. Neoplastic and developmental importance. Dev Biol (NY 1985). 1986; 3:33-57.

46. Taylor DD, Homesley HD, Doellgast GJ. "Membrane-associated" immunoglobulins in cyst and ascites fluids of ovarian cancer patients. Am J Reprod Immunol. 1983; 3:7-11. [PubMed: 6859379]

47. Wang ET, Sandberg R, Luo S, Khrebtukova I, Zhang L, Mayr C, Kingsmore SF, Schroth GP, Burge CB. Alternative isoform regulation in human tissue transcriptomes. Nature. 2008; 456:470476. [PubMed: 18978772] 
48. Winter SF, Minna JD, Johnson BE, Takahashi T, Gazdar AF, Carbone DP. Development of antibodies against p53 in lung cancer patients appears to be dependent on the type of p53 mutation. Cancer Res. 1992; 52:4168-4174. [PubMed: 1322237]

49. Yakirevich E, Sabo E, Lavie O, Mazareb S, Spagnoli GC, Resnick MB. Expression of the MAGEA4 and NY-ESO-1 cancer-testis antigens in serous ovarian neoplasms. Clin Cancer Res. 2003; 9:6453-6460. [PubMed: 14695148] 

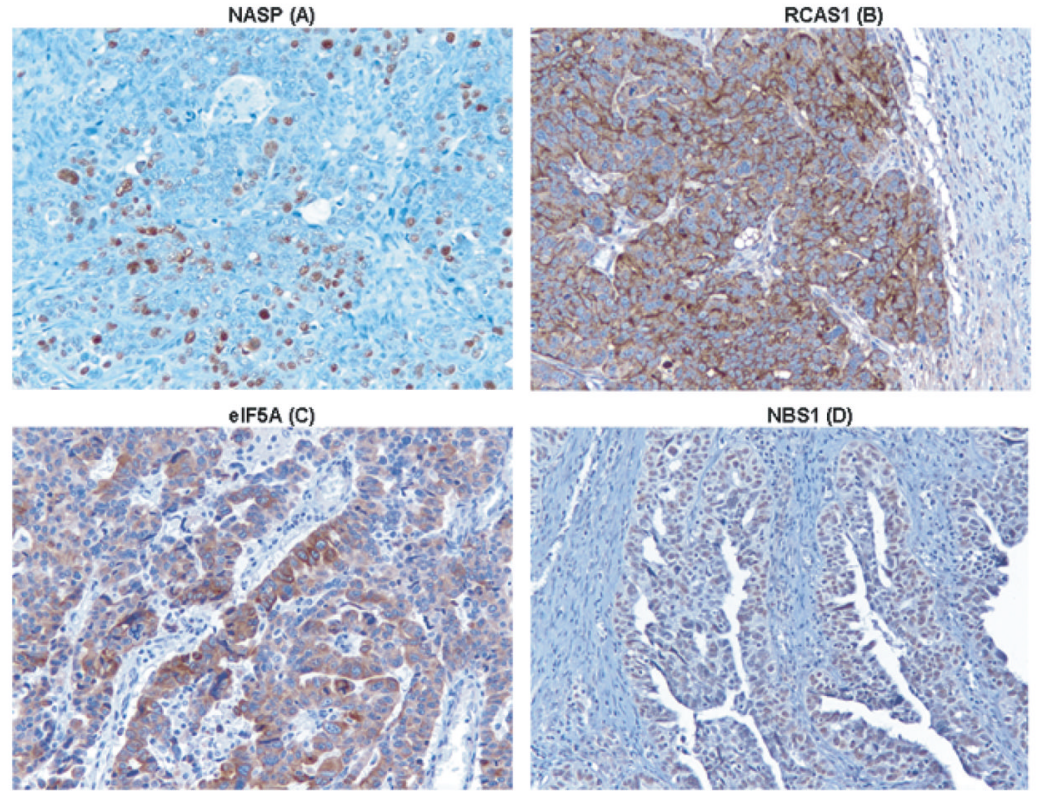

Fig. 1.

Immunohistochemical staining for NASP, RCAS1, eIF5A and NBS1 in tissue sections obtained from a patient with serous high grade carcinoma. IHC was performed to determine the protein expression level of 4 serological biomarkers (mentioned above) that were previously identified by profiling humoral immune responses in OVCA patients [6]. Nuclear staining was observed for NASP (A), cytoplasmic staining was observed for RCAS1 (B) and eIF5A (C), nuclear staining was observed for NBS1 (D). Magnification in all panels is X20. 
MRE11 (A)

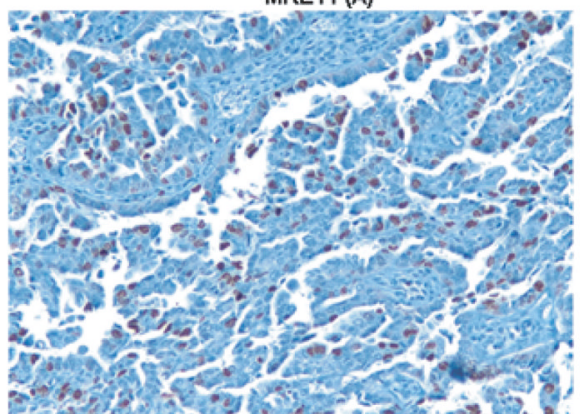

Her2 (C)

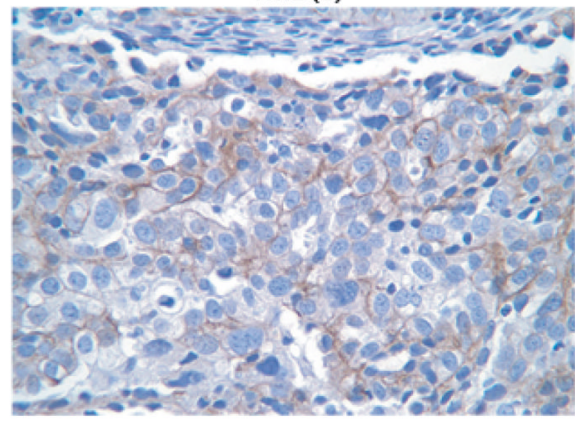

RAD60 (B)

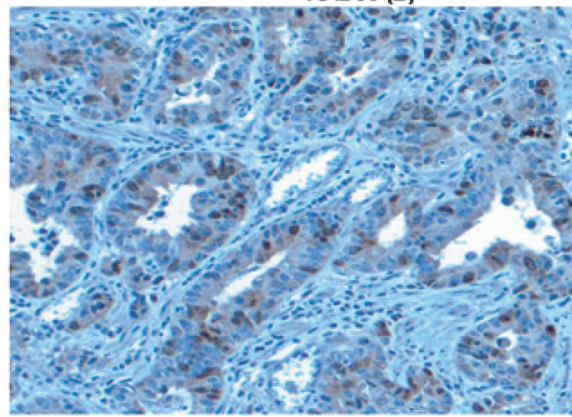

p63 (D)

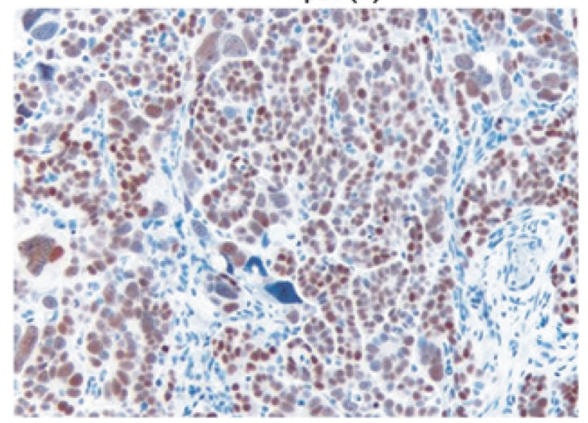

Fig. 2.

Immunohistochemical staining for MRE11, Her2, and p53 in tissue sections obtained from a patient with serous high grade carcinoma. Immunohistochemical staining for RAD50 was performed in tissue sections obtained from a patient with mixed epithelial carcinoma.

Although MRE11 and RAD50 were not previously identified as serological biomarkers, IHC was performed to determine their protein expression level because of their biological functions as stated earlier. Known prognostic markers Her2 and p53 were also included in this study. Nuclear staining was observed for MRE11 (A) and RAD50 (B), membranous staining was observed for Her2 (C), nuclear staining was observed for p53 (D). Magnification in all panels is X20. 

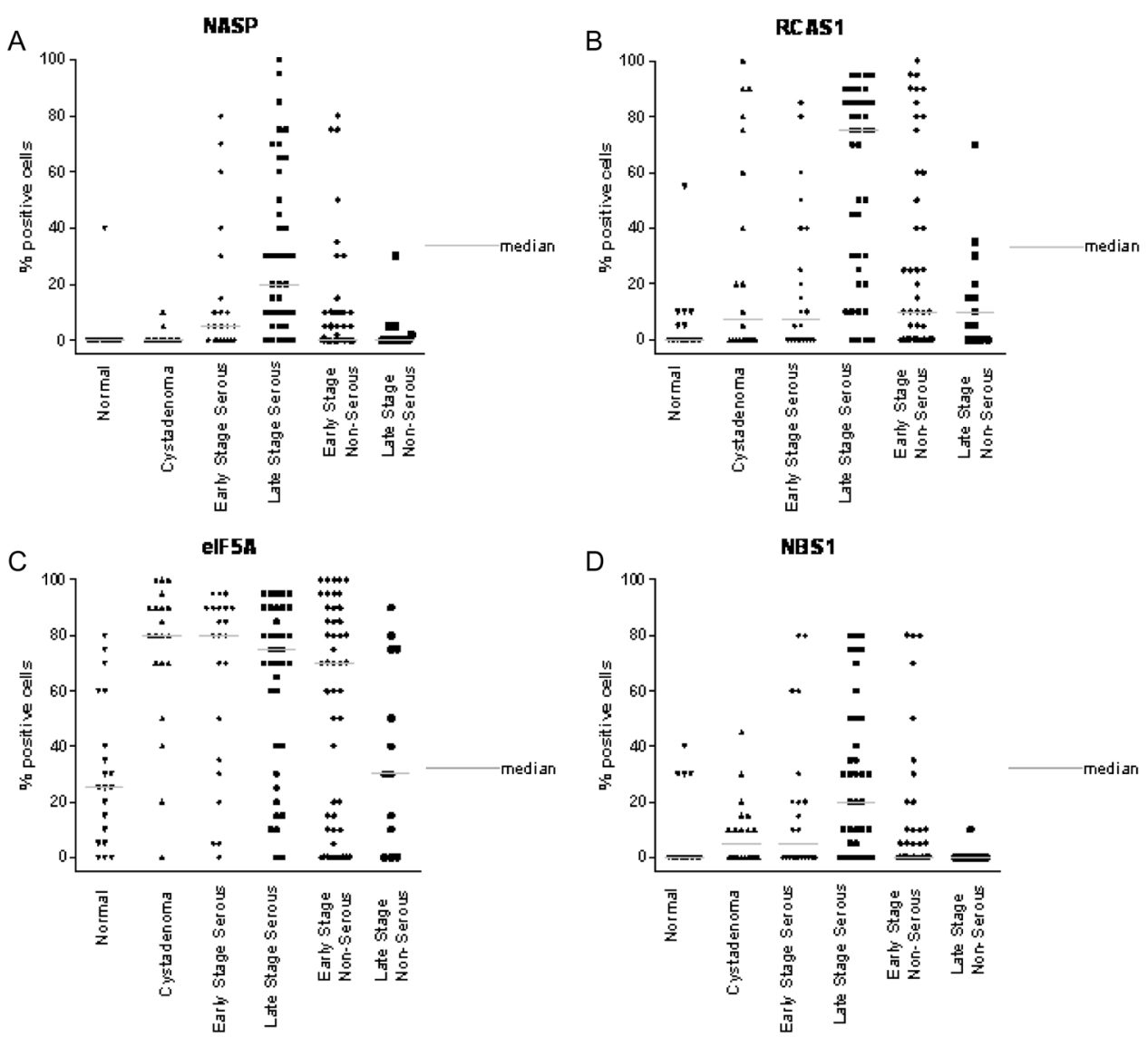

Fig. 3.

Graphical representation of protein expression level obtained by IHC as the percent of cells positively staining for each antigen biomarker. The protein expression of NASP (A),

RCAS1 (B), eIF5A (C) and NBS1 (D) in normal (healthy women), serous cystadenoma, early and late stage serous OVCA, early and late stage non-serous OVCA is shown above. The gray bar indicates the median value for each class of tissue sample. 

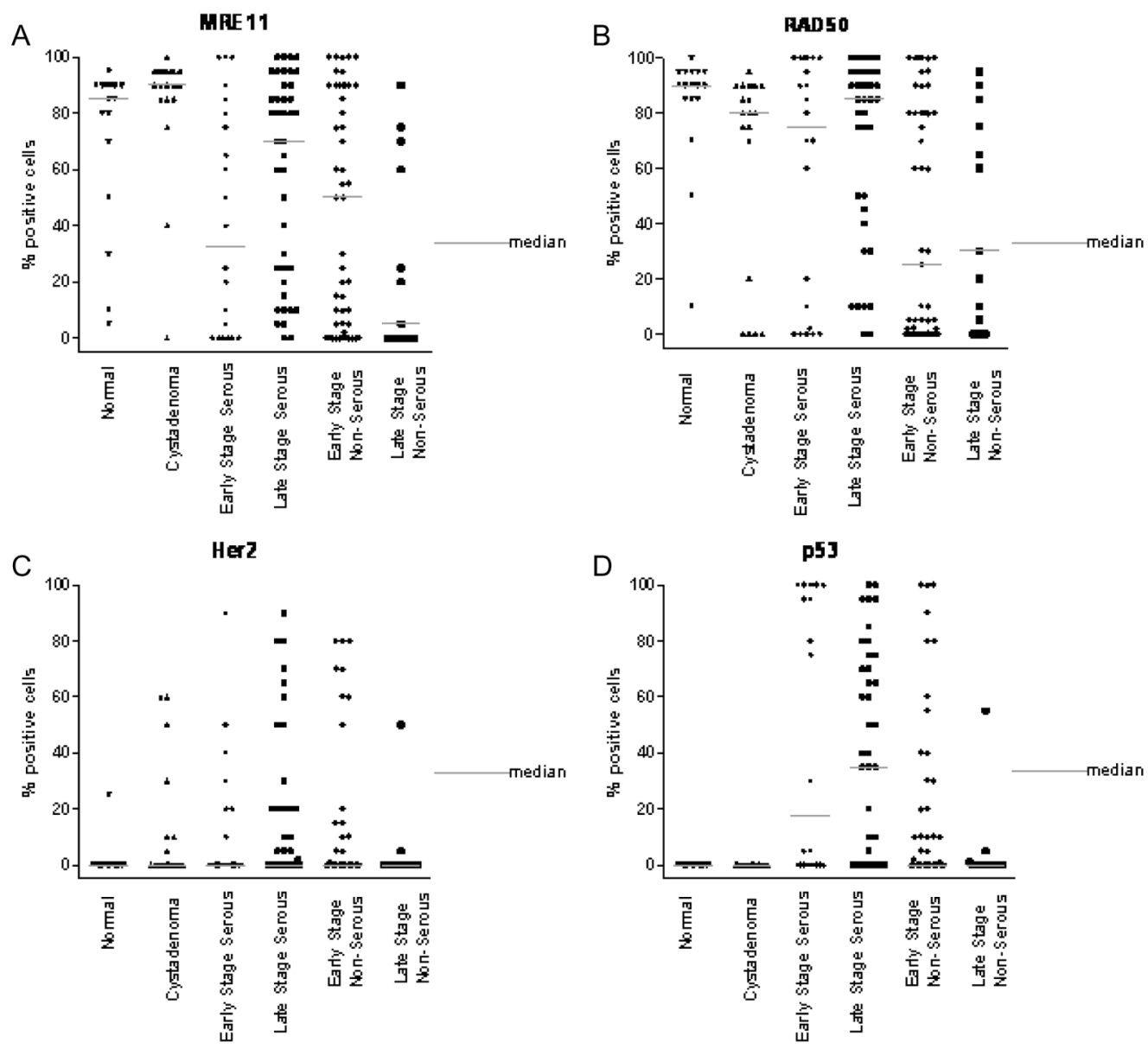

Fig. 4.

Graphical representation of protein expression level of biomarkers obtained by IHC as the percent of cells positively staining for each antigen biomarker The protein expression of MRE11 (A), RAD50 (B) and 2 known biomarkers Her2 (C), and p53 (D) in normal (healthy women), serous cystadenoma, early and late stage serous OVCA, early and late stage nonserous OVCA is shown above. The gray bar indicates the median value for each class of tissue sample. 

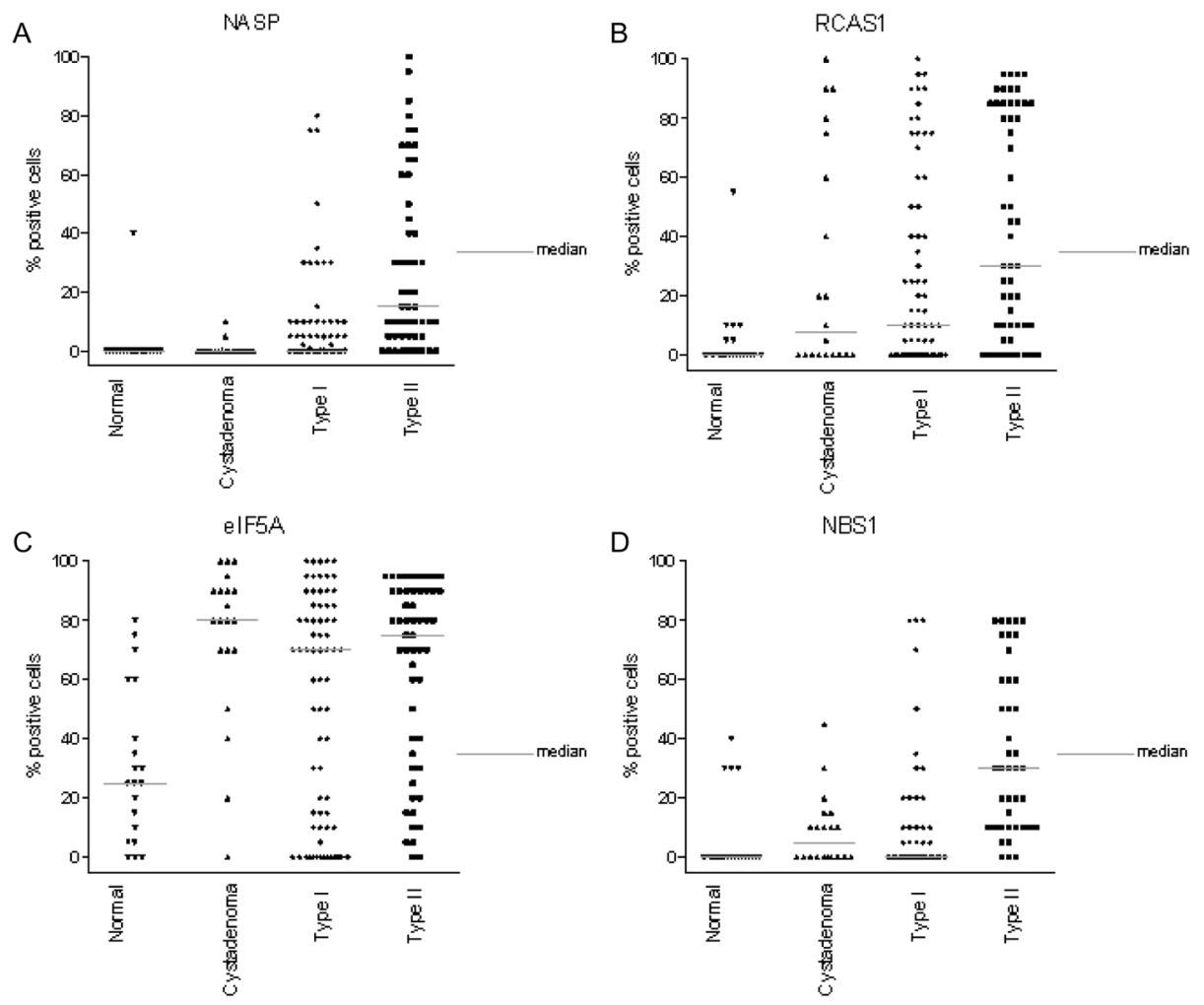

Fig. 5.

Graphical representation of protein expression level of serological biomarkers in Type I and Type II OVCA obtained by IHC as the percent of cells positively staining for each antigen biomarker. The protein expression of NASP (A), RCAS1 (B), eIF5A (C) and NBS1 (D) in normal (healthy women), serous cystadenoma, Type I and Type II OVCA (see Materials and Methods) is shown above. The gray bar indicates the median value for each class of tissue sample. 

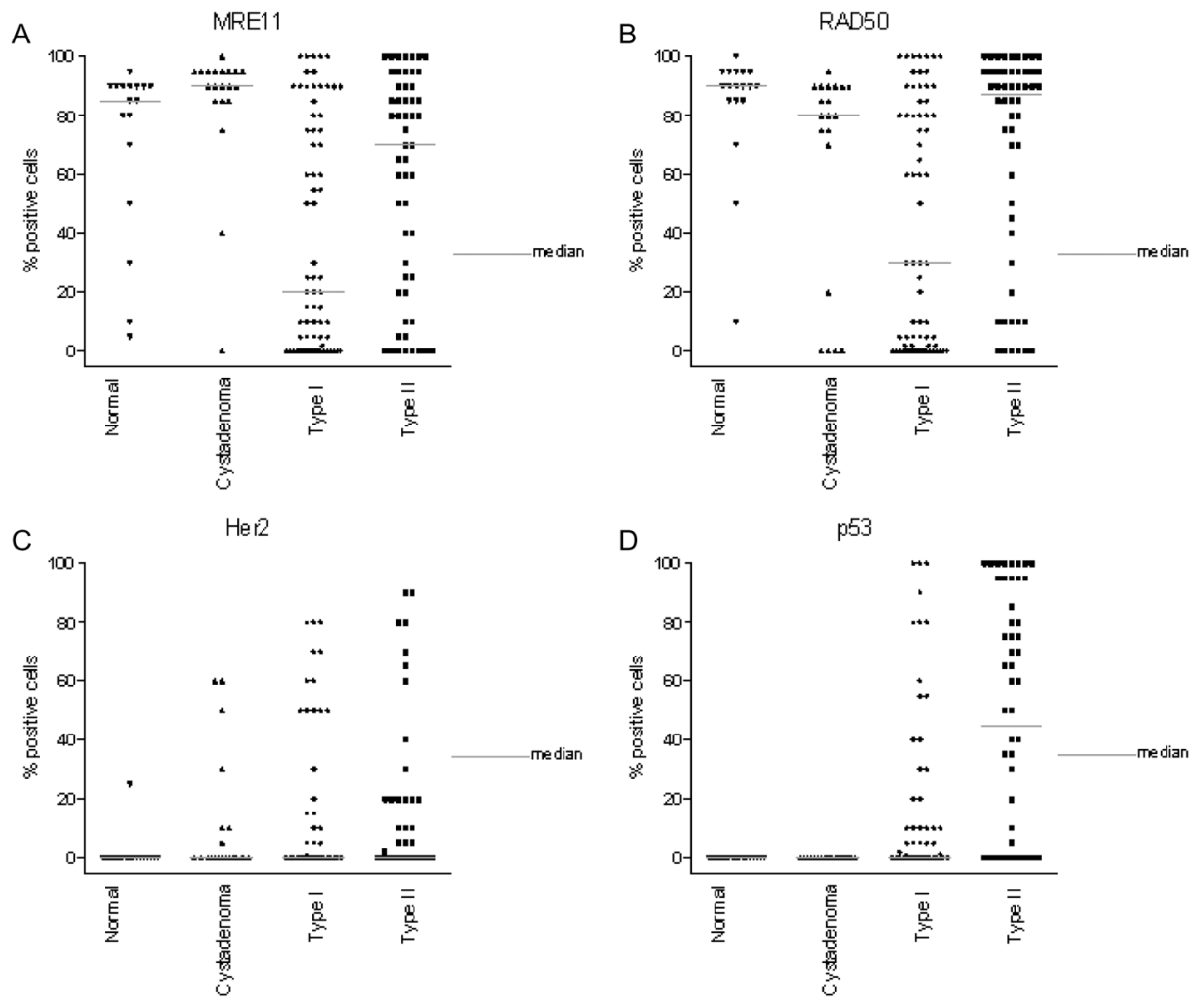

Fig. 6.

Graphical representation of protein expression level of other biomarkers in Type I and Type II OVCA obtained by IHC as the percent of cells positively staining for each antigen biomarker. The protein expression of MRE11 (A), RAD50 (B) and 2 known biomarkers Her2 (C), and p53 (D) in normal (healthy women), serous cystadenoma, Type I and Type II OVCA (see Materials and Methods) is shown above. The gray bar indicates the median value for each class of tissue sample. 


\section{Table 1A}

Age

\begin{tabular}{lccc}
\hline & $\begin{array}{c}\text { OVCA } \\
\text { N=126 }\end{array}$ & $\begin{array}{c}\text { Serous cystadenoma } \\
\mathbf{N = 2 0}\end{array}$ & $\begin{array}{c}\text { Normal } \\
\mathbf{N = 2 0}\end{array}$ \\
\hline Cohort I & & & \\
Age [Median (Interquartile Range)] & $57(49,67)$ & $52(42,66)$ & $49(44,60)$ \\
Range & $27-87$ & $31-89$ & $41-67$ \\
Cohort II & & & \\
Age [Median (Interquartile Range)] & $58(48,69)$ &.-- &.-- \\
Range & $20-89$ & & \\
\hline
\end{tabular}


